\title{
Ventricular Tachycardia Triggered by Loperamide and Famotidine Abuse
}

\author{
Timothy R. Larsen ${ }^{1,2} \cdot$ Jedediah McMunn $^{1} \cdot$ Hala Ahmad $^{1} \cdot$ Soufian T. AlMahameed $^{1}$
}

Published online: 17 February 2018

(c) The Author(s) 2018. This article is an open access publication

\begin{abstract}
A 32-year-old male developed recurrent ventricular tachycardia after taking mega doses of loperamide and famotidine in order to experience an opiate-like euphoric effect. He was taking up to $200 \mathrm{mg}$ of loperamide and multiple doses of famotidine each day. He developed palpitations and syncope. Electrocardiography demonstrated ventricular tachycardia and QT interval prolongation (corrected QT interval was $597 \mathrm{~ms}$ ). He was diagnosed with loperamide-induced QT prolongation resulting in incessant ventricular tachycardia. Loperamide was discontinued, and he was treated with electrolyte replacement, supportive care, and monitoring. After 5 days, his electrocardiogram (ECG) normalized and he had no more ventricular tachycardia. A Naranjo assessment score of 8 was obtained, indicating a probable relationship between QT prolongation and his use of loperamide. Large doses of loperamide can cause QT interval prolongation and lifethreatening arrhythmias. These effects may be accentuated when histamine-2 receptor blockers are also abused.
\end{abstract}

Timothy R. Larsen

Tlarsen17@gmail.com

1 Carilion Clinic, Virginia Tech Carilion School of Medicine, 2001 Crystal Spring Ave, Suite 203, Roanoke, VA 24014, USA

2 Virginia Commonwealth University School of Medicine, PO Box 980509, Richmond, VA 23298, USA

\section{Key Points}

Loperamide toxicity can cause lethal cardiac arrhythmias.

Co-administration of histamine-2 antagonists may increase the opioid-like euphoric effect of loperamide and increase the risk of cardiotoxicity.

Multiple cardiac ion channels may be affected by loperamide overdose.

\section{Introduction}

Loperamide and famotidine are widely available over-thecounter medications. Loperamide is an effective anti-diarrheal agent. It is a synthetic opiate that affects mu-opiate receptors in the gastrointestinal tract to slow intestinal peristalsis and increase rectal tone [1]. At therapeutic doses loperamide does not cross the blood-brain barrier and thus typically does not cause central nervous system effects, including the euphoric effect associated with centrally acting opiates. Recently, there have been several reports of loperamide toxicity from ingestion of supra-therapeutic doses to obtain an opiate-induced euphoric effect $[2,3]$.

In 2016, the United States Food and Drug Administration issued a black box warning regarding serious adverse cardiac effects of loperamide [4]. Loperamide is metabolized by cytochrome P450 3A4 (CYP3A4). Famotidine is a histamine-2 receptor antagonist that, unlike other similar drugs (such as cimetidine), has not demonstrated CYP3A4 
inhabitation at therapeutic doses. It is possible that at supra-therapeutic doses, famotidine does inhibit CYP3A4 (like cimetidine). This inhibition would cause an increase in loperamide serum levels. Herein, we report a case of recurrent ventricular tachycardia (VT) in a patient taking large recreational doses of both loperamide and famotidine.

\section{Case}

A 32-year-old male presented with severe palpitations and syncope. He had a history of prior alcohol, opiate, and methamphetamine abuse, but otherwise no known medical conditions. He admitted to taking, over the prior 10 days, up to $200 \mathrm{mg}$ daily of loperamide (maximum recommended dosage $16 \mathrm{mg}$ per day) and multiple doses of famotidine daily (estimated up to $500 \mathrm{mg}$ per day). Both medications were obtained over the counter. He did not take any other medications, supplements, or recreational substances.

His initial vital signs were as follows: blood pressure 121/76 mmHg; pulse 62 beats/min; temperature $98 \mathrm{~F}$; and oxygen saturation $97 \%$, breathing ambient air. He appeared disheveled, but otherwise had no abnormal physical exam findings. His presenting electrocardiogram (ECG) showed sinus tachycardia at 101 beats/min, first-degree atrioventricular (AV) block, nonspecific intraventricular conduction delay, and prolonged QT interval (Fig. 1). Serum electrolytes, blood counts, cardiac troponin (I), renal, liver, and thyroid function were normal. Transthoracic echocardiogram showed normal chamber size and function and normal valves.

Shortly after admission, he had recurrent symptoms (syncope preceded by palpitations) that occurred during sustained monomorphic VT (Fig. 2). VT terminated spontaneously. Loperamide and famotidine were discontinued, and he was treated with intravenous magnesium and supportive care. During hospitalization, he had frequent multifocal premature ventricular complexes and runs of both monomorphic and polymorphic VT. After 5 days, his PR interval, QRS duration, and QT interval normalized and VT resolved. He was discharged and strongly encouraged to abstain from recreational drug use. At 6 months followup, he had maintained abstinence from recreational drugs and his ECG continued to be normal (Fig. 3).

\section{Discussion}

The findings of incessant VT along with transient ECG abnormalities (first-degree AV block, nonspecific intraventricular conduction delay, and QT prolongation) in a

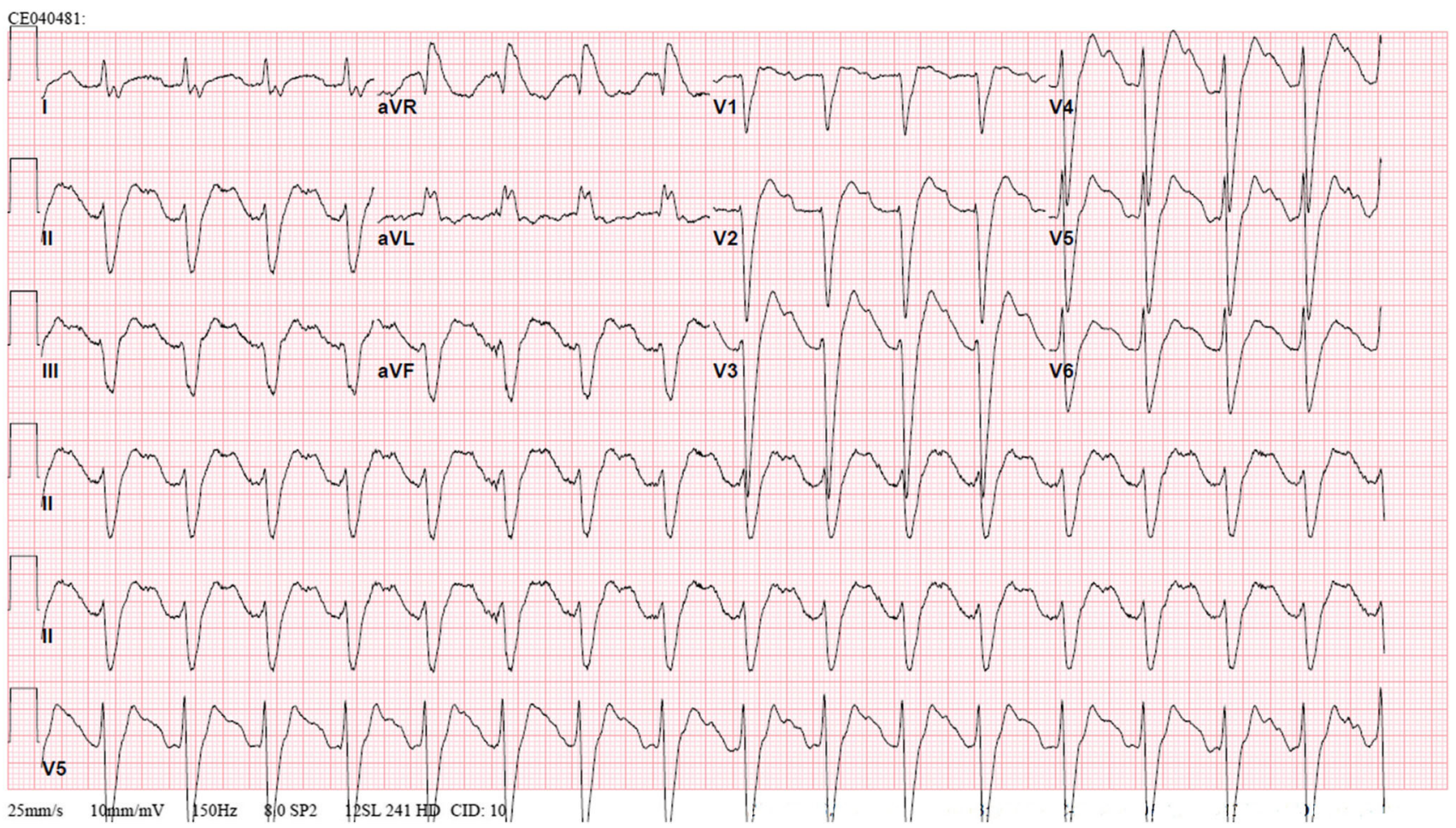

Fig. 1 Electrocardiogram (ECG) demonstrating sinus tachycardia (rate 101 beats/min), first-degree AV block (PR interval $255 \mathrm{~ms})$, nonspecific intraventricular conduction delay (QRS duration $158 \mathrm{~ms}$ ), and QT interval prolongation (corrected QT interval $597 \mathrm{~ms}$ ) 


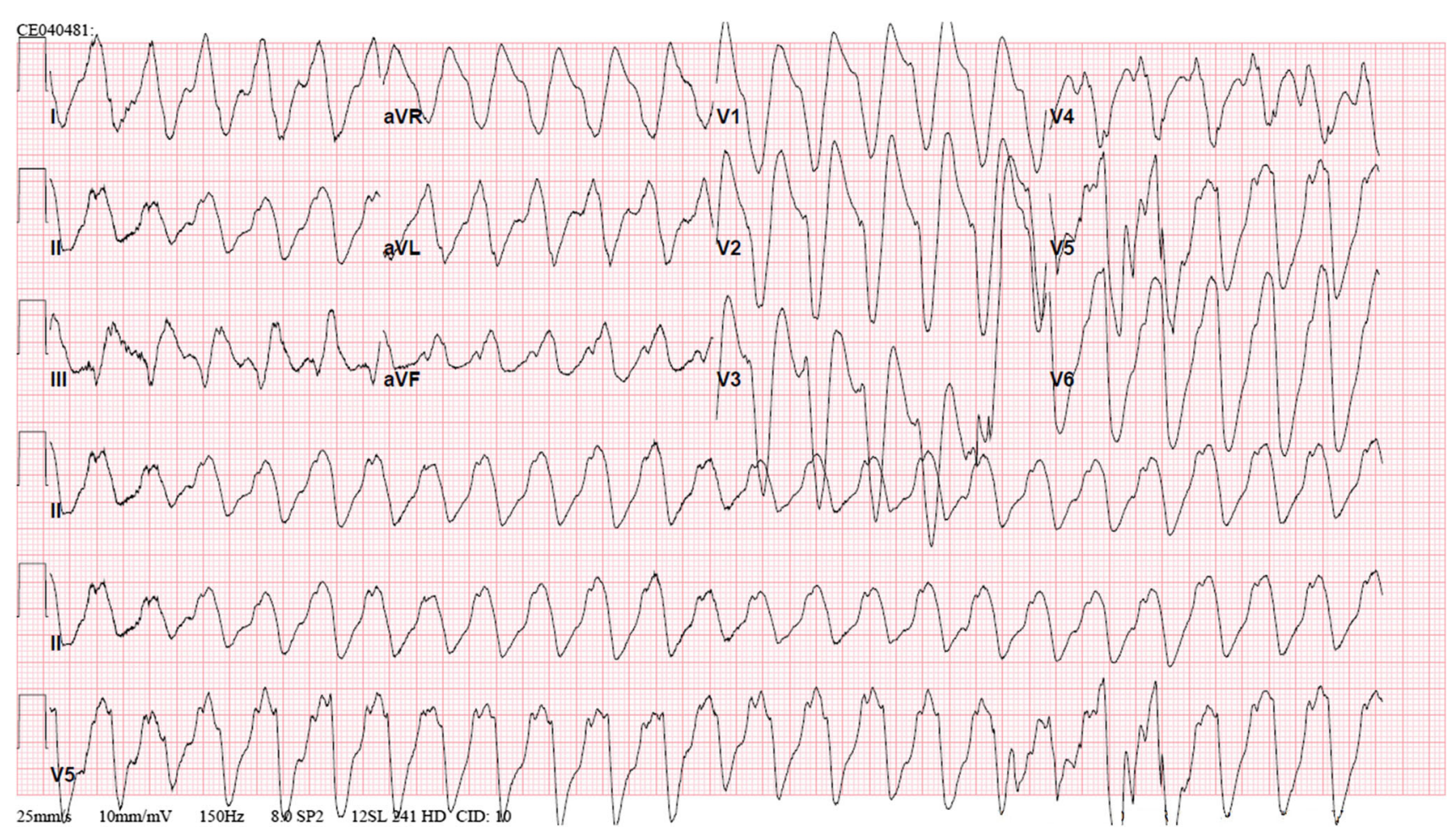

Fig. 2 Electrocardiogram (ECG) demonstrating a monomorphic ventricular tachycardia rate of 150 beats/min

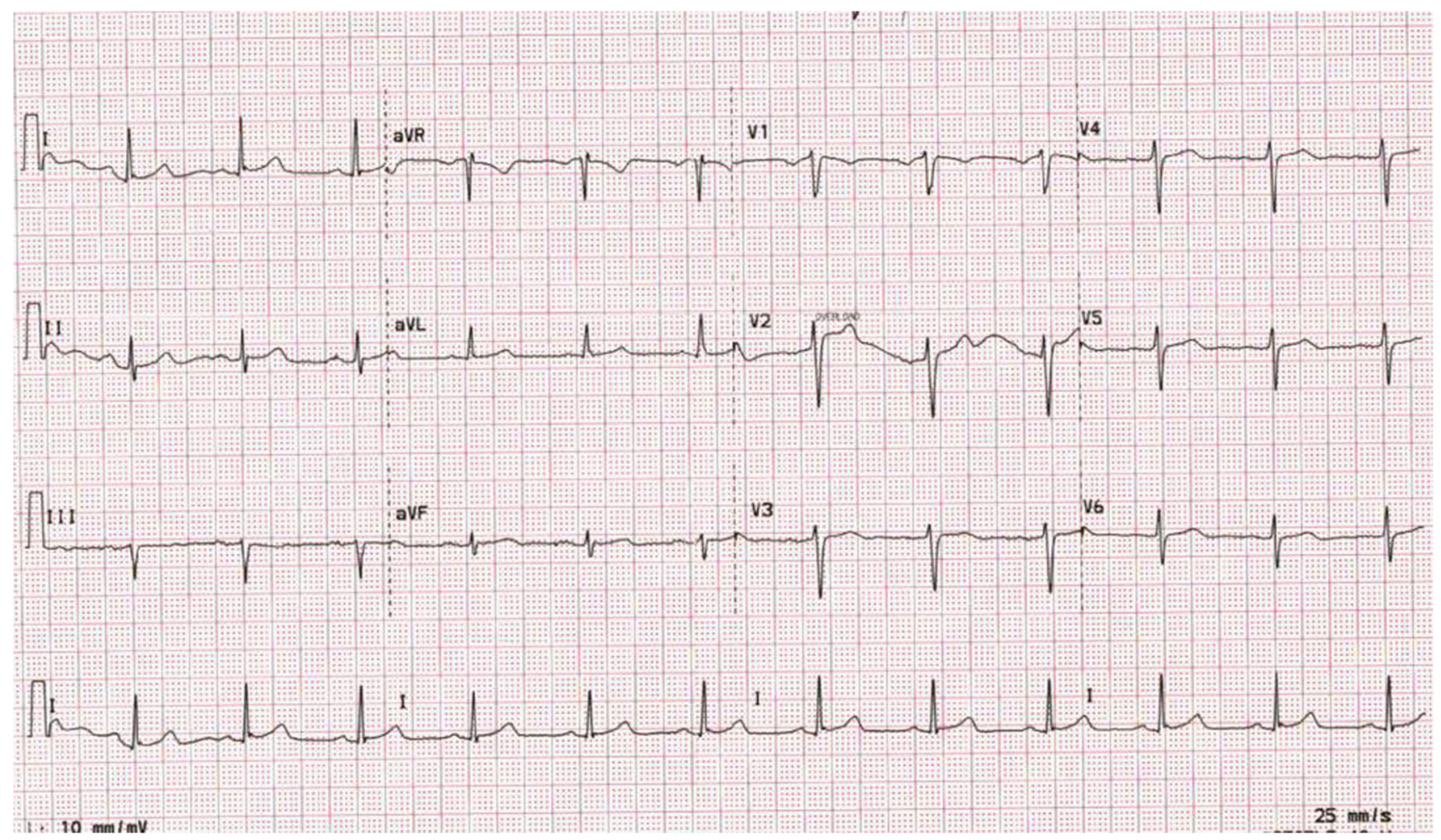

Fig. 3 Electrocardiogram (ECG) demonstrating sinus rhythm (rate 72 beats/min), normal PR interval (188 ms), normal QRS duration (90 ms), and normal QT interval (corrected QT interval $389 \mathrm{~ms}$ ) 
recreational drug user strongly suggest drug toxicity. In this case, the Naranjo assessment score was 8, indicating a probable relationship between QT prolongation and his use of loperamide. Typical symptoms of loperamide overdose are similar to opiate overdose, including central nervous system depression, respiratory depression, and constipation (paralytic ileus). The exact mechanism of loperamide-induced cardiotoxicity is unknown, but it is likely related to alterations in cardiac ion currents and action potential duration (reflected on the ECG as increased QRS duration and QT interval prolongation). QT interval prolongation has been reported with massive loperamide overdose [5].

There is some evidence that loperamide affects L-type calcium channels $\left(I_{\mathrm{CaL}}\right)$ as well as potassium channels- the delayed inward rectifier potassium current $\left(I_{\mathrm{Kr}}\right)$ [6]. Block of these channels results in increased action potential duration and causes QT prolongation (class III and IV antiarrhythmic drug effect). Additionally, QRS widening suggests that loperamide may affect cardiac sodium channels. Blockade of these channels slows action potential upstroke (class I effect), which is responsible for increased QRS duration. A unique aspect of our case is that our patient experienced both sustained monomorphic VT and polymorphic VT. Polymorphic VT is likely due to the QT interval prolonging effect. Monomorphic VT, on the other hand, is not typically due to QT prolongation. The monomorphic VT, in this case, may be a result of drug effect on other cardiac ion channels (possibly sodium channels).

Loperamide undergoes significant first pass metabolism by cytochrome CYP3A4 [7]. High concomitant doses of famotidine may inhibit CYP3A4 (this effect has been demonstrated for other histamine-2 receptor antagonistsparticularly cimetidine) [8-10]. Under these circumstances, the combined effect would be increased serum loperamide levels and enhanced loperamide effects.

Treatment, as with most intoxications, involves discontinuation of the offending agent(s) and supportive care until the toxins are metabolized and cleared from circulation. Naloxone (a competitive inhibitor of central nervous system mu-opioid receptors) is useful for the treatment of the central nervous system effects of loperamide overdose [11]. Naloxone is unlikely to reverse cardiotoxic effects as these are likely mediated by alterations in cardiac ion channel activity (as opposed to an effect of opiate receptor activation). There is no specific antidote for the cardiotoxic effects of loperamide overdose. The loperamide prescribing information claims that activated charcoal can reduce absorption by up to ninefold, and thus should be given in acute overdose. Supportive care includes electrolyte replacement (particularly potassium and magnesium), heart rate support (isoproterenol or temporary overdrive pacing) for bradycardia sensitive polymorphic VT, avoidance of other QT prolonging agents and defibrillation.
Funding No sources of funding were received for the preparation of this article.

\section{Compliance with Ethical Standards}

Conflict of interest Timothy Larsen, Jedediah McMunn, Hala Ahmad, and Soufian AlMahameed report no conflicts of interest relevant to the content of this article.

Informed consent Written informed consent was obtained from the patient for publication of this case report. A copy of the written consent may be requested for review from the corresponding author.

Open Access This article is distributed under the terms of the Creative Commons Attribution-NonCommercial 4.0 International License (http://creativecommons.org/licenses/by-nc/4.0/), which permits any noncommercial use, distribution, and reproduction in any medium, provided you give appropriate credit to the original author(s) and the source, provide a link to the Creative Commons license, and indicate if changes were made.

\section{References}

1. Read M, Read NW, Barber DC, Duthie HL. Effects of loperamide on anal sphincter function in patients complaining of chronic diarrhea with fecal incontinence and urgency. Dig Dis Sci. 1982;27(9):807-14.

2. Mukarram O, Hindi Y, Catalasan G, Ward J. Loperamide induced Torsades de Pointes: a case report and review of the literature. Case Rep Med. 2016;2016:4061980.

3. Marzec LN, Katz DF, Peterson PN, Thompson LE, Haigney MC, Krantz MJ. Torsade de Points associated with high-dose loperamide ingestion. J Innov Card Rhythm Manag. 2015;6:1897-9.

4. FDA Drug Safety Communication: FDA warns about serious heart problems with high dose of the antidiarrheal medicine loperamide (Imodium), including from abuse and misuse. https:// www.fda.gov/Drugs/DrugSafety/ucm504617.htm. Accessed 6 July 2016.

5. Leung G, Altshuler D, Goldenberg R, Fridman D, Yuriditsky E. Conduction disturbances and ventricular arrhythmias associated with high-dose loperamide. J Clin Toxicol. 2016;6:309.

6. Yang SB, Major F, Tietze LF, Rupnik M. Block of delayedrectifier potassium channels by reduced haloperidol and related compounds in mouse cortical neurons. J Pharm Exp Ther. 2005;315(1):352-62.

7. Kim KA, Chung J, Jung DH, Park JY. Identification of cytochrome $\mathrm{P} 450$ isoforms involved in the metabolism of loperamide in human liver microsomes. Eur $\mathrm{J}$ Clin Pharmacol. 2004;60:575-81.

8. Rendic S, Di Carlo FJ. Human cytochrome P450 enzymes: a status report summarizing their reactions, substrates, inducers, and inhibitors. Drug Metab Rev. 1997;29(1-2):413-580.

9. O'Connell CW, Schricker AA, Schneir AB, Metushi IG, Birgersdotter-Green U, Minns AB. High-dose loperamide abuse-associated ventricular arrhythmias. Heart Rhythm Case Rep. 2016;2(3):232-6.

10. Lasoff DR, Schneir AS. Ventricular dysrhythmias from loperamide misuse. J Emerg Med. 2016;50(3):508-9.

11. Friedli G, Haenggeli CA. Loperamide overdose managed by naloxone. Lancet. 1980;1(8183):1413. 